\title{
DGNR: eFellowship geht 2022 in die zweite Runde
}

Nach dem Erfolg der ersten Staffel bietet die DGNR seit Januar 2022 erneut ein 6-monatiges eFellowship Interventionelle Neuroradiologie an. Dabei nehmen junge Neuroradiologinnen und Neuorradiologen mithilfe eines ferngesteuerten, hochwertigen Kamerasystems mit Sprech- und Tonverbindung aus der Ferne live bei spannenden Neurointerventionen teil und können ihren erfahrenen DGNR-Mentorinnen und -Mentoren im wahrsten Sinne des Wortes über die Schulter schauen.
Dies ermöglicht während aller Eingriffe, die live übertragen werden, eine „Eins-zu-eins“Kommunikation mit der behandelnden Interventionalistin/dem behandelnden Interventionalisten und eine direkt anschließende Diskussion der Fälle.

Das Ziel des eFellowship Interventionelle Neuroradiologie ist es, jungen Neuroradiologinnen und Neuroradiologen die gesamte Bandbreite neurointerventioneller Verfahren zu vermitteln, die von sehr erfahrenen

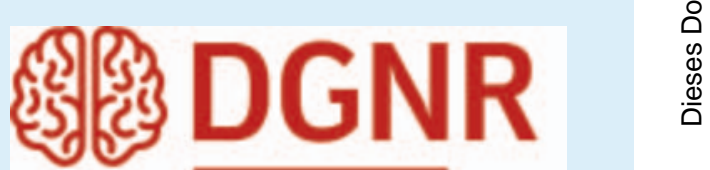

eFELLOWSHIP INTERVENTIONELLE NEURORADIOLOGIE

Neurointerventionalistinnen und -interventionalisten durchgeführt werden. 
Die eFellows erlangen theoretische Expertise in allen Aspekten der interventionellen Neuroradiologie, unter anderem in der Behandlung des akuten ischämischen Schlaganfalles, zerebralen Aneurysmen, arteriovenösen Malformationen des Gehirns und der Wirbelsäule oder von angeborenen zerebralen und spinalen Fehlbildungen.

Das Programm wird begleitet von interaktiven Falldiskussionen und virtuellen Meetings und soll den intensiven fachlichen
Austausch über die Grenzen der Institute hinaus fördern.

Weitere Informationen zum eFellowship finden Sie unter www.dgnr.de 